\title{
Papillary Thyroid Carcinoma Variants
}

\author{
Ricardo V. Lloyd · Darya Buehler • \\ Elham Khanafshar
}

Received: 5 November 2010/Accepted: 13 December 2010/Published online: 8 January 2011

(C) Springer Science+Business Media, LLC 2010

\begin{abstract}
Papillary thyroid carcinomas are the most common thyroid cancers and constitute more than $70 \%$ of thyroid malignancies. The most common etiologic factor is radiation, but genetic susceptibility and other factors also contribute to the development of papillary thyroid carcinoma. The most common variants include conventional, follicular variant and tall cell variant. However, many other uncommon variants have been described including oncocytic, columnar cell, diffuse sclerosing and solid forms. Immunohistochemical staining with TTF-1 and thyroglobulin is very useful in confirming the diagnosis of papillary thyroid carcinoma especially in metastatic sites. Markers such as HBME-1 and CITED1 can assist in separating some difficult cases of follicular variants of papillary thyroid carcinomas from follicular adenomas. Molecular studies have shown that the BRAF V600E mutation is found mainly in papillary and anaplastic thyroid carcinomas. Other molecular markers such as HMGA2 and insulin-like growth factor II mRNA binding protein 3 have been used recently as molecular tests to separate papillary thyroid carcinoma and its variants from follicular adenomas and other benign thyroid nodules.
\end{abstract}

Keywords Papillary thyroid carcinoma $\cdot$ Follicular variant · Tall cell · Columnar cell · BRAF mutation . RET/PTC $\cdot$ HMGA2

R. V. Lloyd $(\bowtie) \cdot$ D. Buehler

Department of Pathology and Laboratory Medicine, University of Wisconsin School of Medicine and Public Health, 600 Highland Avenue, K4/436 CSC, Madison, WI 53792, USA e-mail: rvlloyd@wisc.edu

E. Khanafshar

Department of Pathology, University of California School of Medicine, San Francisco, CA, USA

\section{Introduction}

Papillary thyroid carcinoma (PTC) is the most common type of malignant thyroid tumor constituting more than $70 \%$ of thyroid malignancies $[1,2]$. There has been an increasing incidence of PTC worldwide for the past few decades. There are significant geographic variations, but sampling techniques also contribute to this wide variability.

The etiology of PTC is related to environmental, genetic and hormonal factors [1-3]. Radiation as a genotoxic factor for PTC has been well documented [1, 2]. The widespread use of X-irradiation in the 1950s for treating conditions such as enlarged thymuses and acne have contributed to the increased incidence of PTC [2]. The Chernobyl nuclear accident in 1986 led to a marked increase in the incidence of PTC in Belarus and other areas of the former Soviet Union close to the accident [2]. The most striking increase in the incidence was in children, since the growth of the thyroid is most rapid in childhood and falls to low levels in adults [2].

Patients with PTC usually present with a cold nodule on radioactive iodine scan [1,2]. A few patients may present with cervical lymphadenopathy. Involvement of the Delphian lymph node is an adverse prognostic sign in PTC, since this may indicate advanced disease with a need to examine the central and lateral lymph node compartments more carefully [4].

Patients with PTC usually present with a palpable nodule in the thyroid. Non-palpable nodules of PTC may be discovered incidentally after CT and MRI examination. Small nodules of PTC or microcarcinomas (less than $1 \mathrm{~cm}$ in size) are usually of no clinical significance especially in young patients (less than 40 years), since such individuals have a 20 -year survival of greater than $98 \%$ even with palpable tumors. 
Fine needle aspiration biopsy (FNAB) is the most common method of examining solitary thyroid nodules today. FNAB of enlarged cervical lymph nodes may also reveal a PTC, since patients may present with a palpable cervical LN even when the primary tumor is small or nonpalpable.

The gross appearance of PTC can be quite variable. Most tumors are firm solid and gray-white, but a significant percentage of tumors may be cystic. It is not uncommon to have a solid primary tumor with a cystic metastasis to a lymph node. PTC may have an infiltrative growth pattern in the thyroid or may show direct extension beyond the thyroid to adjacent tissues.

The conventional PTC shows a papillary architecture with branching $[1,2]$. The papillae are covered by cells with eosinophilic cytoplasm and enlarged nuclei (Fig. 1a). The polarity of the cells may be abnormal or lost in some tumors. Squamous metaplasia may be present. Psamomma bodies with concentric lamellae composed partly of thyroglobulin are more common in some variants of PTC. Some tumors may also contain multinucleated giant cells.

Cytologic features are very helpful in making the diagnosis in some variants of PTC. These consist of enlarged irregular nuclei that are often oval shaped and
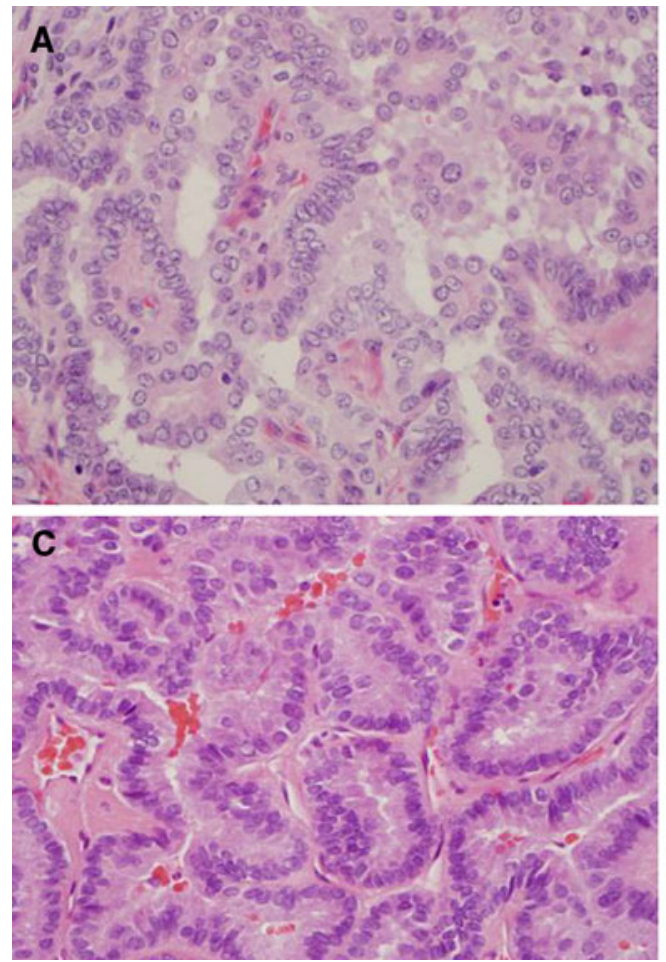

Fig. 1 Conventional papillary thyroid carcinoma and papillary thyroid carcinoma variants. a Conventional papillary thyroid carcinoma. The carcinoma has papillae with fibrovascular cores and enlarged nuclei with nuclear clearing. b Follicular variant of papillary thyroid carcinoma. The follicles are made up of cells with enlarged nuclei with cytologic features of papillary carcinoma. The colloid is overlapping because of the nuclear enlargement. The nuclei often show clearing or have a ground glass appearance with prominent nuclear grooves and pink cytoplasmic invaginations $[1,2]$.

\section{Discussion}

There are numerous histopathologic variants of PTC (Table 1, Figs. 1a, b, c, d and 2a, b, c, d). Each variant shows a combination of specific growth patterns, cell types and stromal changes. A major problem in classifying PTC into various subtypes is that the criteria used to define these subtypes are not rigorously defined, so different pathologist may not agree with these subtype classifications. There is general agreement that the subtype classification that is used should constitute the predominant pattern of the neoplasm.

\section{Follicular Variant}

These tumors look like follicular neoplasm when examined grossly. They are composed of follicles of variable sizes. The colloid is usually darker or hypereosinophilic
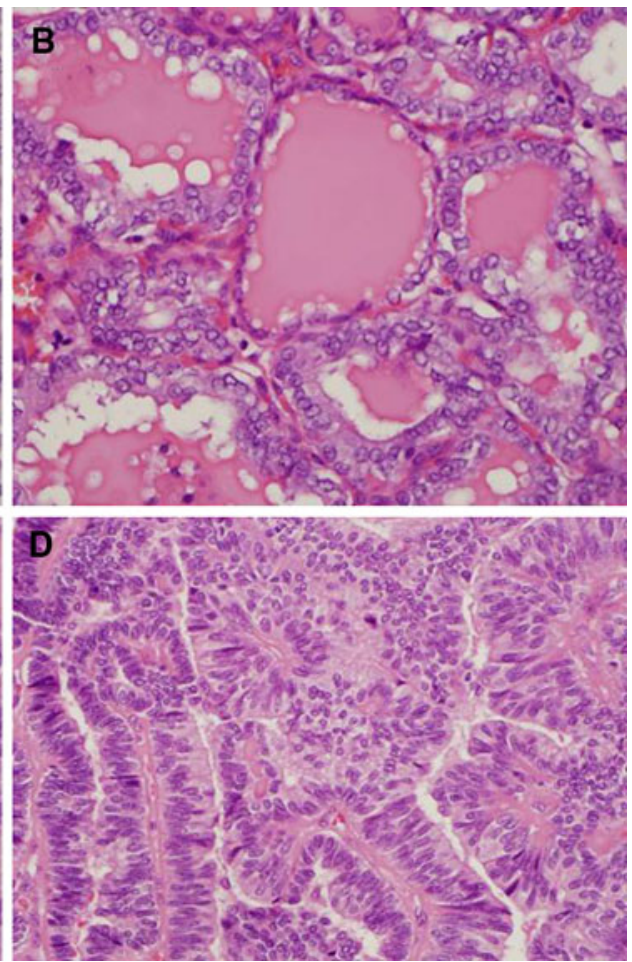

darker and shows scalloping. $\mathbf{c}$ Tall cell variant of papillary thyroid carcinoma. The cells are 2-3 times as tall as they are wide and show cytologic features of papillary thyroid carcinoma. d Columnar cell variant of papillary thyroid carcinoma. The pseudostratified cells have enlarged nuclei which are overlapping 
Table 1 Variant of papillary thyroid carcinoma

- Conventional

- Follicular variant

- Papillary microcarcinoma

- Tall cell

- Oncocytic

- Columar cell

- Diffuse sclerosing

- Solid

- Clear cell

- Cribriform morular

- Macrofollicular

- PTC with prominent hobnail features

- PTC with fasciitis-like stroma

- Combined papillary and medullary carcinoma

- PTC with dedifferenatiation to anaplastic carcinoma

compared to the colloid in adjacent non-neoplastic thyroid and may show scalloping "bubble gum" appearance (Fig. 1b). Occasional multinucleated giant cells are present within the follicles. The cytological features of PTC are important to establish the diagnosis in these tumors $[1,2]$.
The diagnosis of follicular variant of PTC can be quite difficult and controversial [5, 6]. The prognosis of these tumors is similar to the typical PTC. An exception is the diffuse or multinodular follicular variant, which has a more aggressive clinical course [2]. The prognosis of follicular variant of PTC also depends on whether they are completely encapsulated or invasive $[2,7]$.

Follicular variant of PTC can look like a follicular neoplasm except for the cytological features. Because these tumors can be easily confused with follicular adenomas and follicular carcinomas, the use of immunohistochemical and molecular markers can be very useful in confirming the diagnosis in difficult cases as will be discussed below.

\section{Papillary Microcarcinoma}

These tumors are usually found incidentally and measure less than $1 \mathrm{~cm}$ in diameter. Patients may occasionally present with cervical lymph node metastases. The tumors are often close to the thyroid capsule. When they are nonencapsulated and show extensive sclerosis they may be more aggressive than completely encapsulated tumors $[1,2]$.
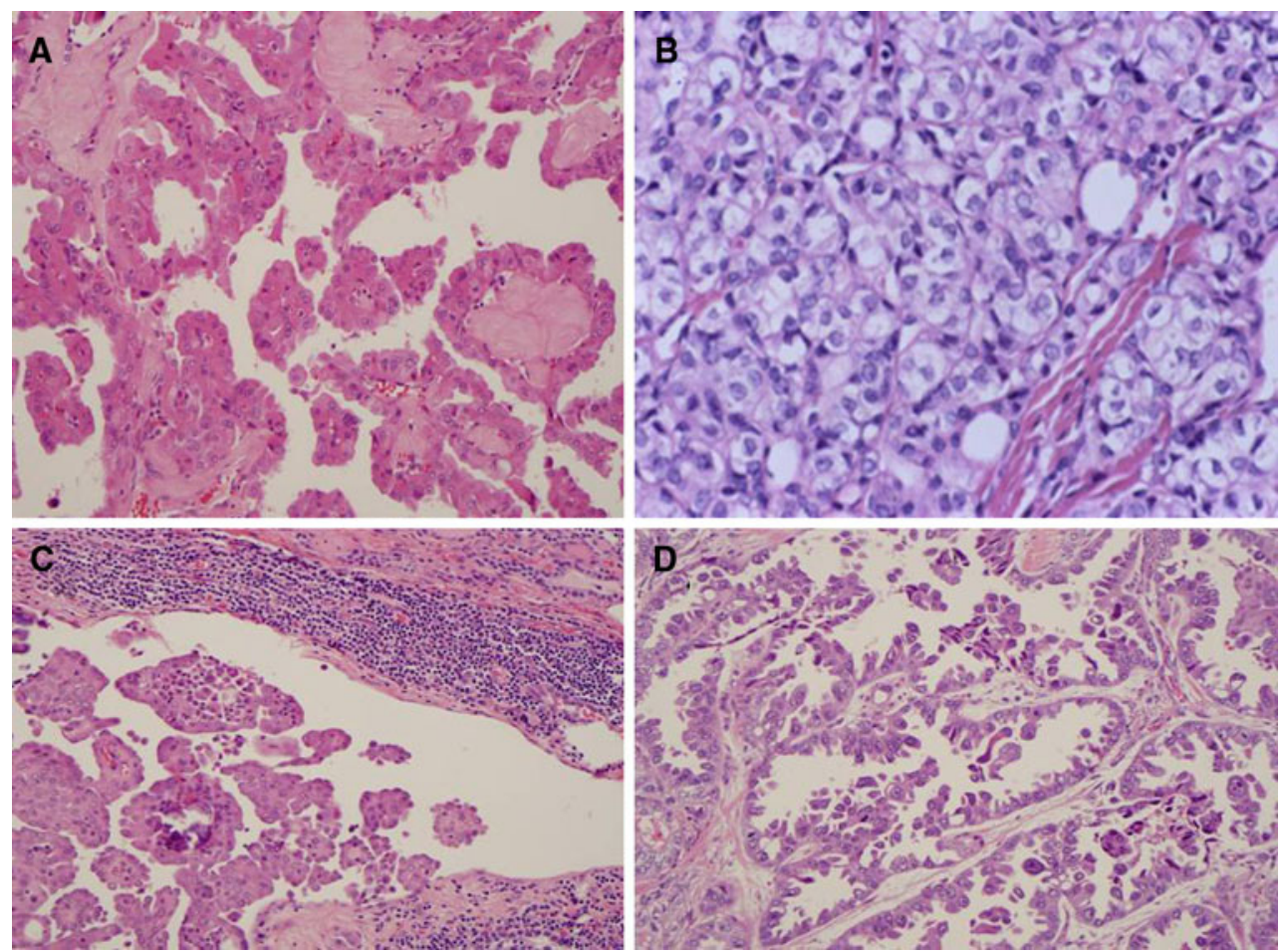

Fig. 2 Variants of papillary thyroid carcinoma. a Oncocytic variant with large cells showing abundant eosinophilic cytoplasm and nuclear features of papillary thyroid carcinoma. b Solid variant with sheets of tumor cells with abundant cytoplasm and nuclear features of papillary thyroid carcinoma including cytoplasmic invagination into nuclei. c Diffuse sclerosing variant showing abundant psammoma bodies,

and squamoid changes in the tumor cells. A prominent lymphocytic infiltrate is present in the background. d Papillary thyroid carcinoma with prominent hobnail features. The tumor consists of hobnail cells with loss of polarity and high grade cytologic features including prominent nucleoli and increased mitotic activity 


\section{Tall Cell Variant}

This variant is composed of cells whose height is at least 2-3 times as tall as they are wide. The tumor cells have abundant eosinophilic cytoplasm and nuclear features similar to conventional PTC (Fig. 1c). Nuclear pseudoinclusions may be more prominent. The tumors tend to be larger than the conventional PTCs, so necrosis, mitotic activity and extrathyroidal extensions are more common. Most patients are older and present with large bulky tumors and they usually have a more aggressive course than the usual PTC $[1,2,8,9]$.

\section{Oncocytic Variant}

These tumors have a distinct brown color on gross examination similar to the follicular Hurthle cell tumors. They may have follicular or papillary architecture [10] (Fig. 2a). The "Warthin-like" oncocytic tumor has abundant lymphocytic stromal infiltrate and is often seen with Hashimoto's thyroiditis $[2,15]$. The diagnosis is usually based on the nuclear features of PTC.

\section{Columnar Cell Variant}

This is a rare variant that is made up of pseudo stratified columnar cells. Some cells may have supranucleur and subnuclear cytoplasmic vacuoles. Some tumors may resemble endometrial or colonic adenocarcinomas [11] (Fig. 1d). The nuclear features of conventional PTC are not well represented in these tumors, so if the patient presents with metastatic lesions, they may be mistaken for metastatic adenocarcinoma from lung, colon or endometrium. However, the tumor cells are usually positive for TTF-1 and they are variably positive for thyroglobulin $[1,2]$. Although these tumors have been considered by some authors to be more aggressive than the typical PTC, this finding has been more controversial. As with many PTC, columnar cell variant tumors that are completely encapsulated are less likely to metastasize.

\section{Diffuse Sclerosing Variant}

These tumors are more common in younger patients between 15 and 30 years of age [12]. They are characterized by diffuse involvement of the thyroid and both lobes are typically involved. Papillary structures in dilated lymphovascular spaces are often present. The tumors show extensive squamous metaplasia, abundant psamomma bodies, stromal fibrosis and prominent lymphocytic infiltration (Fig. 2c). The background thyroid often shows chronic lymphocytic thyroiditis. A significant number of patients may have lymph node and lung metastasis, but the tumors do not usually lead to the demise of the patient $[1,2]$.

\section{Solid Cell Variant}

These tumors consist of sheets of tumor cells, which have cytological features of typical PTC [19] (Fig. 2b). Vascular invasion and extrathyroidal extension are present in about a third of cases [2]. They are more common in children with a history of radiation exposure.

\section{Clear Cell Variant}

These tumors comprised of mainly clear cells have a papillary architecture and cytological features of PTC. Some tumors may have oncocytic and clear cell features [2]. Immunostaining for TTF-1 and thyroglobulin may be necessary to distinguish these tumors from a metastatic clear cell carcinoma.

\section{Cribriform-Morular Variant}

This rare variant is usually associated with familial adenomatous polyposis and Gardner Syndrome. The tumor has a prominent cribriform pattern with solid and spindle cell areas as well as squamous morules. Focal papillary architecture is usually present. The nuclei show clearing and grooves focally. The tumors usually involve both thyroid lobes.

There is some debate in the literature as to whether this is a variant of PTC or a distinct lesion [2]. The tumor is positive for thyroglobulin focally and shows positive nuclear staining for beta catenin.

\section{Macrofollicular Variant}

This tumor is extremely uncommon. It is composed largely of macrofollicles and can be easily confused with a hyperplastic or colloid nodules from low power observation. The cytological features of PTC are seen at higher magnification [2]. Metastatic tumors to lymph nodes usually retain the macrofollicular patterns.

\section{Papillary Thyroid Carcinoma with Prominent Hobnail} Features

This rare variant was recently described [13]. The tumors in the reported series usually had more than $30 \%$ of the tumor with hobnail features (Fig. 2d). Tall cell and diffuse sclerosing patterns were present in a small percentage of these tumors in some cases [13]. These are very aggressive carcinomas associated with mortality from metastatic PTC in $50 \%$ of the 8 cases recently reported [13]. The general 
concept of loss of cellular polarity [14] has been used to describe similar type thyroid tumors, which may be related to micropapillary carcinomas in other sites such as breast, pancreas, lung and urinary bladder [15].

Papillary Thyroid Carcinoma with Fasciitis-like Stroma

These are rare variants of PTC associated with fasciitis like stroma or fibromatosis-like stroma. The importance of recognizing this variant is that they should not be confused with anaplastic carcinomas secondary to dedifferentiation of a conventional PTC.

Combined Papillary Thyroid Carcinoma and Medullary Thyroid Carcinoma

This composite tumor consists of separate areas or cells with PTC or medullary thyroid carcinoma [2]. Immunostaining for thyroglobulin and calcitonin helps to make the distinction of the two components.

Papillary Thyroid Carcinoma with Dedifferentiation to Anaplastic Carcinoma

PTC can undergo dedifferentiation or transformation to anaplastic carcinoma [1, 2]. In a study of 109 anaplastic carcinomas, Albores Saavedra et al. found that $46.8 \%$ PTC coexisted with anaplastic carcinoma (34 conventional type 14 tall cell variant and 3 follicular variant supporting the concept of dedifferenation of PTC leading to anaplastic carcinoma [16].

Immunohistochemical Markers in Papillary Thyroid Carcinoma

Immunohistochemical markers such as TTF-1 and thyroglobulin are very helpful in confirming the thyroid origin of PTC, especially when the tumor is present outside of the thyroid gland $[1,2]$ (Table 2). TTF-1 is also expressed in

Table 2 Immunohistochemical markers in papillary thryoid carcinoma

- TTF-1

- Thyroglobulin

- Thyroid peroxidase

- CD56 (NCAM)

- PAX8

- HBME-1

- CITED1

- Cytokeratin 19

- Galectin 3 lung carcinomas and small cell carcinomas in many sites, so it is most useful when combined with thyroglobulin.

Because of difficulties in distinguishing some follicular variants of PTC from adenomas and adenomatoid nodules, markers such as HBME-1 and CITED-1 have been used with increasing frequency [17]. In our experience HBME-1 and CITED 1 are very useful in such settings, but as with most markers, they have to be used with the appropriate histologic and cytologic findings. Other markers such as cytokeratin 19, Galectin 3, CD56, PAX 8 and thyroid peroxidase have not been as useful in these differential diagnosis because of lower sensitivity and specificity $[1,2]$.

\section{Molecular Markers in Papillary Thyroid Carcinoma}

BRAF mutation (V600E) has proven to be relatively restricted to PTC and anaplastic thyroid carcinomas, and is very useful in the differential diagnosis difficult thyroid tumors (Table 3). Unfortunately the follicular variant of papillary thyroid carcinomas has a BRAF mutation in only $5-20 \%$ of the time while conventional PTC shows a BRAF mutation more often (35-70\%) (39-42). BRAF mutations have been reported to be predictive of several factors including tumor behavior and response to radioactive iodine $(43,44)$, but more studies are needed in this area.

RET/PTC rearrangement is found mainly in PTC with a highly variable frequency (5-80\%) in different geographic regions and in different studies [18]. There are different fusions of the tyrosine kinase domain of RET. RET/PTC1 is the most common followed by RET/PTC3. In children with PTC, which developed after exposure to radiation, RET/PTC3 was the dominant rearrangement [18].

Chromosomal rearrangements involving the TRK gene are found in about $10 \%$ of PTC. This results from the fusion of tyrosine kinase domain of TRK on chromosome $1 \mathrm{q} 22$ to the tropomyosin gene [18].

Activating point mutations of RAS proto-oncogene occur in a small percentage of PTC. The N-RAS codon is the most common. Follicular variant of PTC has a higher frequency of RAS mutations than in other PTC. RAS mutations are also present in follicular carcinomas and adenomas [18].

Table 3 Molecular markers in papillary thyroid carcinomas

- BRAF mutation

- RET/PTC rearrangements

- RAS mutations

- TRK rearrangements

- Beta catenin

- HMGA2 overexpression

- Insulin like growth factor II mRNA-binding protein 3 (IMP3) 
HMGA2 and insulin-like growth factor II mRNA binding protein 3 (IMP3) are highly expressed during fetal development and then tissue levels are very low to absent in adult tissues. Recent studies have shown that in many malignant neoplasms HMGA2 and IMP3 have increased levels of expression [19, 20]. These studies have shown that normal thyroid tissues and follicular adenomas express low levels of HMGA2 and IMP3 mRNA compared to papillary and follicular carcinomas. The levels of expression in the carcinomas range from 1.5 to greater that ten fold higher than in adenomas or normal thyroid tissues, so measurements of the levels of expression by quantitative RT-PCR helps to separate benign from low grade malignant thyroid neoplasms in most cases [19, 20]. These observations have been examined in thyroid tissues and molecular assays are being developed to distinguish follicular adenomas from papillary and follicular carcinomas in cytologic specimens as well as in tissues using formalin fixed paraffin embedded tissues. These approaches should allow the pathologist to use molecular approaches in solving difficult diagnostic problems in thyroid pathology.

\section{Conclusions}

Papillary thyroid carcinomas are the most common thyroid malignancies. Some subtypes of PTC such as tall cell and columnar cell variants appear to have a more aggressive biological course. Recent studies of biomarkers such as HBME-1 and CITED 1 suggest that they can be useful in separating follicular variant of PTC from follicular adenomas. Molecular studies have shown that HMGA2 and IMP3 may be useful diagnostically in separating benign and malignant thyroid tumors.

\section{References}

1. Khan A, Nose V. In: Lloyd RV, editor. Endocrine pathology: differential diagnosis and molecular advances, 2nd ed. New York: Springer 2010; p. 181-236.

2. DeLellis RA, Lloyd RV, Heitz PU, Eng C, editors. Pathology and genetics of tumours of endocrine organs. In: Kleihues P, Sobrin LH, series editors. World health organization. Classification of Tumours. Lyon: IARC Press; 2004.

3. Hemminiki K, Li X. Familial risk of cancer by site and histopathology. Int J Cancer. 2003;103:105-9.

4. Isaacs JD, Lundgren CI, Sidhu SB, et al. The Delphian lymph node in thyroid cancer. Ann Surg. 2008;247:477-82.
5. Wallander M, Layfield LJ, Jarboe E, et al. Follicular variant of papillary carcinoma: reproducibility of histologic diagnosis and utility of HBME-1 immunohistochemistry and BRAF mutational analysis as diagnostic adjuncts. Appl Immunohistochem Mol Morphol. 2010;18:231-5.

6. Lloyd RV, Erickson LA, Casey MB, et al. Observer variation in the diagnosis of follicular variant of papillary thyroid carcinoma. Am J Surg Pathol. 2004;28:1336-40.

7. Rivera M, Ricarte-Filho J, Patel S, et al. Encapsulated thyroid tumors of follicular cell origin with high grade features (high mitotic rate/tumor necrosis): a clinicopthologic and molecular study. Hum Pathol. 2010;41:172-80.

8. Hawk WA, Hazard JB. The many appearances of papillary carcinoma of the thyroid. Cleve Clin Q. 1976;43:207-15.

9. Johnson TL, Lloyd RV, Thompson NW, et al. Prognostic implications of the tall cell variant of papillary thyroid carcinoma. Am J Surg Pathol. 1988;12:22-7.

10. Herrera MF, Hay ID, Wu PS, et al. Hurthle cell (oxyphilic) papillary thyroid carcinoma: a variant with more aggressive biologic behavior. World J Surg. 1992;16:669-74.

11. Evans HL. Columnar-cell carcinoma of the thyroid. A report of two cases of an aggressive variant of thyroid carcinoma. Am J Clin Pathol. 1986;85:77-80.

12. Koo JS, Hong S, Park CS. Diffuse sclerosing variant is a major subtype of papillary thyroid carcinoma in the young. Thyroid. 2009;19:1225-31.

13. Asioli S, Erickson LA, Sebo TJ, et al. Papillary thyroid carcinoma with prominent hobnail features: a new aggressive variant of moderately differentiated papillary carcinoma. A clinicopathologic immunohistochemical, and molecular study of eight cases. Am J Surg Pathol. 2010;34:44-52.

14. Kakudo K, Tang W, Ito Y, et al. Papillary carcinoma of the thyroid in Japan: subclassification of common type and identification of low risk group. J Clin Pathol. 2004;10:1041-6.

15. Nassar H. Carcinomas with micropapillary morphology: clinical significance and current concepts. Adv Anat Pathol. 2004;11: 297-303.

16. Albores-Saavedra J, Hernandez M, Sanchez-Sosa S, et al. Histologic variants of papillary and follicular carcinomas associated with anaplastic spindle and giant cell carcinomas of the thyroid: an analysis of rhabdoid and thyroglobulin inclusions. Am J Surg Pathol. 2007;31:729-36.

17. Scognamiglio T, Hyjek E, Kao J, Chen YT. Diagnostic usefulness of HBME1, galectin-3, CK19, and CITED1 and evaluation of their expression in encapsulated lesions with questionable features of papillary thyroid carcinoma. Am J Clin Pathol. 2006;126: $700-8$.

18. Nikiforova MN, Nikiforov YE. Molecular genetics of thyroid cancer: implications for diagnosis treatment and prognosis. Expert Rev Mol Diagn. 2008;8:83-95.

19. Lappinga PJ, Kip NS, Jin L, et al. HMGA2 gene expression analysis performed on cytologic smears to distinguish benign from malignant thyroid nodules. Cancer Cytopathol. 2010;118: 287-97.

20. Jin L, Seys AR, Zhang S, et al. Diagnostic utility of IMP3 expression in thyroid neoplasms: a quantitative RT-PCR study. Diagn Mol Pathol. 2010;19:63-9. 